\title{
MiR-125b regulates proliferation and apoptosis of nasopharyngeal carcinoma by targeting A20/NF-кB signaling pathway
}

\author{
Zhen Zheng ${ }^{1,2}$, Jia-Quan Qu ${ }^{1,3}$, Hong-Mei Yi ${ }^{1,2}, X_{\text {u Ye }}{ }^{1,2}$, Wei Huang ${ }^{1,2}$, Ta Xiao ${ }^{1,4}$, Jiao-Yang Li ${ }^{1,2}$, Yuan-Yuan Wang ${ }^{1,2}$, Juan Feng ${ }^{1,2}$,
} Jin-Feng $\mathrm{Zhu}^{1,2}$, Shan-Shan $\mathrm{Lu}^{1,2}$, Hong $\mathrm{Yi}^{*, 1,2}$ and Zhi-Qiang Xiao ${ }^{*, 1,2}$

MiR-125b is aberrantly expressed and has a role in the various types of tumors. However, the role and mechanism of miR-125b in nasopharyngeal carcinoma (NPC) are unclear. In this study, we investigated the role and mechanism of miR-125b in NPC. We observed that miR-125b was significantly upregulated in the NPC tissues relative to normal nasopharyngeal mucosa (NNM), and its increment was correlated with poor patient survival, and was an independent predictor for reduced patient survival; miR-125b promoted NPC cell proliferation and inhibited NPC cell apoptosis; in a mouse model, administration of miR-125b antagomir significantly reduced the growth of NPC xenograft tumors. Mechanistically, we confirmed that A20 was a direct target of miR-125b, and found that activation of nuclear factor $\kappa$ B (NF- $\kappa B$ ) signaling pathway by A20 mediated miR-125b-promoting NPC cell proliferation and -inhibiting NPC cell apoptosis. With a combination of loss-of-function and gain-of-function approaches, we further showed that A20 inhibited NPC cell proliferation, induced NPC cell apoptosis, and reduced the growth of NPC xenograft tumors. Moreover, A20 was significantly downregulated, whereas p-p65(RelA) was significantly upregulated in the NPC tissues relative to normal nasopharyngeal mucosa, and miR-125b level was negatively associated with $A 20$ level, whereas positively associated with p-p65 level. Our data demonstrate that miR-125b regulates NPC cell proliferation and apoptosis by targeting A20/ NF- $\kappa B$ signaling pathway, and miR-125b acts as oncogene, whereas A20 functions as tumor suppressor in NPC, highlighting the therapeutic potential of miR-125b/A20/NF-KB signaling axis in the NPC.

Cell Death and Disease (2017) 8, e2855; doi:10.1038/cddis.2017.211; published online 1 June 2017

MicroRNAs (miRNAs) are a class of non-coding RNAs that post-transcriptionally silence target mRNAs. ${ }^{1}$ MiRNAs have an important role in normal cell homeostasis, and dysregulation of miRNAs expression has been implicated in human cancers. $^{2,3}$ One miRNA that has gained special interest in the field of cancer research is miR-125b. ${ }^{4}$ In some tumor types, miR-125b is upregulated and displays oncogenic potential, as it induces cell proliferation and inhibits cell apoptosis, contributing to malignant transformation..$^{5-7}$ In other tumors, miR-125b is downregulated and accompanied by derepression of cellular proliferation and anti-apoptotic programs, corresponding to the loss of tumor suppressive functions. ${ }^{5-7}$ Although miR-125b is aberrantly expressed in a great variety of tumors and acts as oncogene or tumor suppressor, the expression and function of miR-125b in nasopharyngeal carcinoma (NPC) are unclear.

Nuclear factor $\kappa \mathrm{B}(\mathrm{NF}-\kappa \mathrm{B})$ is an important regulator of proliferation and apoptosis. ${ }^{8}$ Activation of NF-KB signaling pathway has a crucial role in the development and progression of NPC. ${ }^{9-12}$ NF-kB comprises two subunits, commonly p50 (NFKB1)/p65(RelA), which in its inactive state is held in the cytoplasm by the inhibitor of NF-KB $\left(I_{\kappa} \mathrm{B}\right)$. $I_{\kappa} \mathrm{B}$ is regulated by $I_{\kappa} \mathrm{B}$ kinases (IKKs): upon stimulation by external signals or stress, IKK is activated and phosphorylates $I_{\kappa} \mathrm{B}$, and thereby targets $I_{K} \mathrm{~B}$ to ubiquitin-mediated protein degradation. As a result, NF- $\kappa \mathrm{B}$ is released and translocates into the nucleus where it transactivates multiple genes involved in proliferation and apoptosis. 8,13

Ubiquitin modification, occurring at multiple steps within the $\mathrm{NF}-\kappa \mathrm{B}$ signaling cascades, serves as a regulator in NF- $\mathrm{BB}$ activation. ${ }^{14} \mathrm{~A}$ growing number of proteins, such as receptorinteracting protein kinase (RIP-1), TRAF2, TRAF6, and $\mathrm{NEMO}$, in the NF- $\kappa \mathrm{B}$ signal pathway have been identified to be modified by ubiquitin. ${ }^{14-17}$ Tumor necrosis factor alphainduced protein 3 (TNFAIP3, also known as A20), functioning as an ubiquitin-editing enzyme, ${ }^{15}$ negatively regulates NF- $\mathrm{B}$ signaling through dual mechanisms, that is, deconjugation of K63-linked polyubiquitin chains from RIP-1 and subsequent conjugation of RIP-1 with K48-linked polyubiquitin chains for proteasomal degradation. ${ }^{16,17}$ A20 can also catalyze the cleavage of K63-linked ubiquitin chains and the conjugation of K48-linked polyubiquitin chains, thereby targeting TRAF2, TRAF6 and NEMO for proteasomal degradation. ${ }^{18,19}$ Therefore, A20 serves as a negative regulator in NF- $\kappa \mathrm{B}$ signal pathway by inhibiting the activity of its upstream signaling transducers.

\footnotetext{
${ }^{1}$ Research Center of Carcinogenesis and Targeted Therapy, Xiangya Hospital, Central South University, Changsha, Hunan 410008, China; ${ }^{2}$ The Higher Educational Key Laboratory for Cancer Proteomics and Translational Medicine of Hunan Province, Xiangya Hospital, Central South University, Changsha, Hunan 410008, China; ${ }^{3}$ Department of Oncology, Qianjiang Central Hospital of Chongqing, Jishou University, Hunan 416000, China and ${ }^{4}$ Department of Dermatology, Xiangya Hospital, Central South University, Changsha, Hunan 410008, China

${ }^{*}$ Corresponding author: H Yi or Z-Q Xiao, Research Center of Carcinogenesis and Targeted Therapy, Xiangya Hospital, Central South University, Changsha 410008, Hunan China. Tel: +86 731 89753378; Fax: +86 731 84327332; E-mail: yi_hong@126.com or zqxia02001 @ hotmail.com

Received 26.1.17; revised 09.4.17; accepted 10.4.17; Edited by E Candi
} 
Several studies have reported that miR-125b activates NF- $\kappa$ B pathway by targeting A20. ${ }^{20-22}$ Therefore, we hypothesize that miR-125b is dysregulated in NPC, and has a crucial role in the pathogenesis of NPC by targeting A20 and then regulating NF-kB signaling activity. Here, we report that miR-125b is upregulated, whereas A20 is downregulated in NPC tissues relative to normal nasopharyngeal mucosa (NNM); miR-125b promotes NPC cell proliferation and inhibits NPC cell apoptosis by targeting A20/NF-кB signaling pathway; A20 inhibits NPC cell proliferation, induces NPC cell apoptosis, and reduces the growth of NPC xenograft tumors. Our data demonstrate that miR-125b acts as oncogene, whereas A20 functions as tumor suppressor in NPC, and suggest that NPC patients with high miR-125b expression might benefit from specific targeted therapies directed at miR-125b/A20/NF-kB signaling pathway.

\section{Results}

MiR-125b expression is increased in the NPC tissues and correlated with NPC patient prognosis. As the expression and significance of miR-125b in NPC are unclear, we detected miR-125b levels in the NPC tissues and NNM by using qRT-PCR. We observed that miR-125b expression was significantly increased in the NPC tissues as compared with NNM (Figure 1a). Survival analyses revealed that high miR-125b level in NPC tissues correlated with the markedly reduced disease-free survival (DFS) and overall survival (OS) of the patients (Figure 1b). A univariate and multivariate Cox regression analysis showed that high miR-125b expression was an independent predictor for the reduced DFS and OS of NPC patients (Supplementary Table S1). These results indicate the importance of high miR-125b expression in the NPC.

MiR-125b promotes NPC cell proliferation and inhibits NPC cell apoptosis. We used paired NPC cell lines, CNE2IR and CNE2, to determine the effect of miR-125b on NPC cell proliferation and apoptosis. CNE2-IR cell line was derived from NPC CNE2 cell line, both cell lines have the same genetic background but different radiosensitivity, and CNE2$\mathrm{IR}$ is radioresistant, whereas CNE2 is radiosenstive. ${ }^{23}$ Because miR-125b expression was significantly higher in the CNE2-IR cells than that in the CNE2 cells (Figure 2a), miR-125b inhibitor and mimic were transfected into CNE2-IR and CNE2 cells, respectively, and then cell proliferation was determined by cell counting Kit-8 (CCK-8) assay, 5-ethynyl2 '-deoxyuridine (EdU) incorporation and plate colony formation assay. The results showed that miR-125b inhibitor significantly decreased, whereas miR-125b mimic significantly increased NPC cell proliferation as compared with control inhibitor or mimic (Figures $2 b-d$ ). Next, we analyzed the effect of miR-125b on the apoptosis of NPC cells by using flow cytometry. The results showed that miR-125b inhibitor significantly increased while miR-125b mimic significantly decreased the apoptosis of NPC cells as compared with control inhibitor or mimic (Figure 2e). Collectively, these

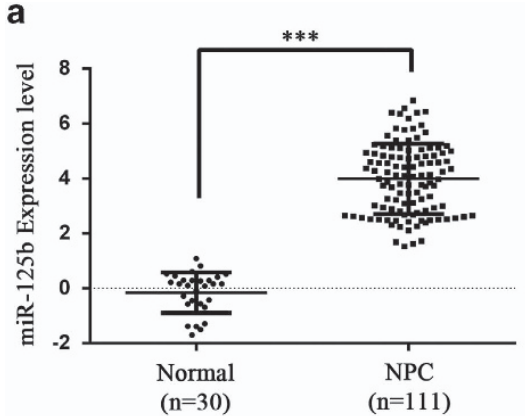

b

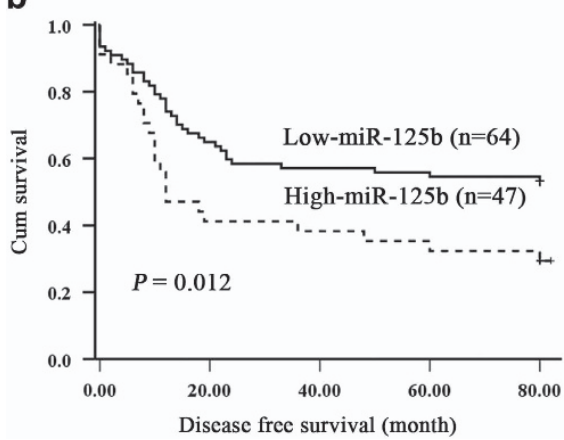

C
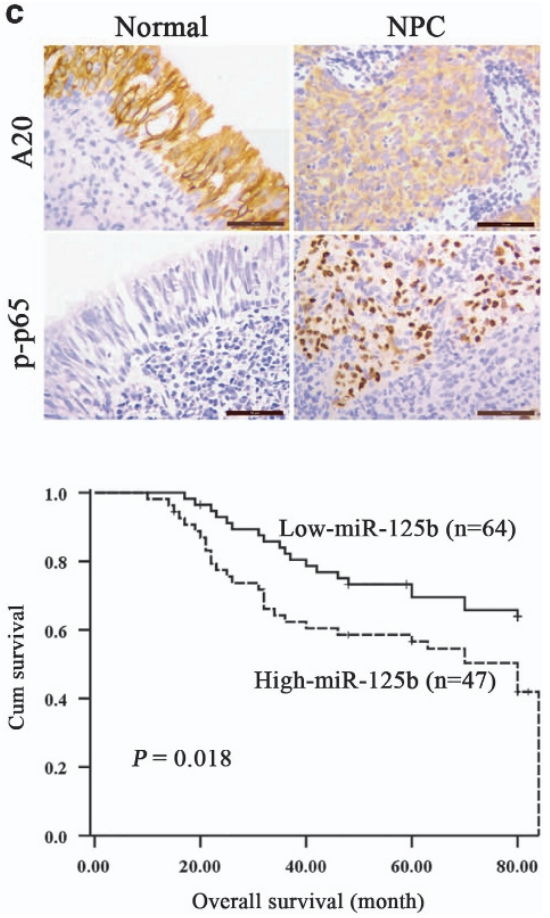

Figure 1 The expression of miR-125b, A20, and p-p65 in NPC and association of miR-125b expression levels with the patient survival. (a) QRT-PCR analysis of the expression levels of miR-125b in the $111 \mathrm{NPC}$ tissues and 30 normal nasopharyngeal mucosa (NNM). Three experiments were done; Means, S.D.s, and statistical significance are denoted; ${ }^{* * *} P<0.001$. (b) Kaplan-Meier survival analysis for 111 NPC patients according to the expression levels of miR-125b. NPC patients with high miR-125b expression have a significantly worse disease-free survival (left) and overall survival (right) than those with low miR-125b expression. The log-rank test was used to calculate $P$-value. (c) A representative result of immunohistochemistry showing the expression of A20 and p-p65 (RelA) in the NNM and NPC tissues. Scale bars $=50 \mu \mathrm{m}$ 
a

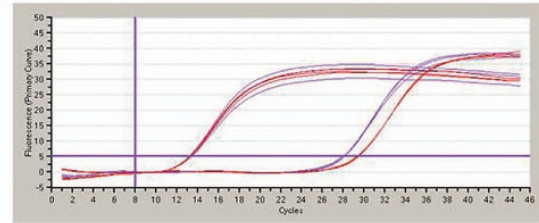

miR-125b amplification curve

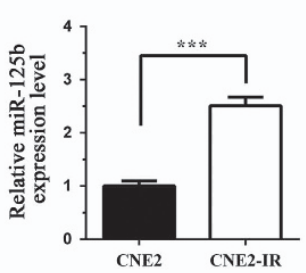

d
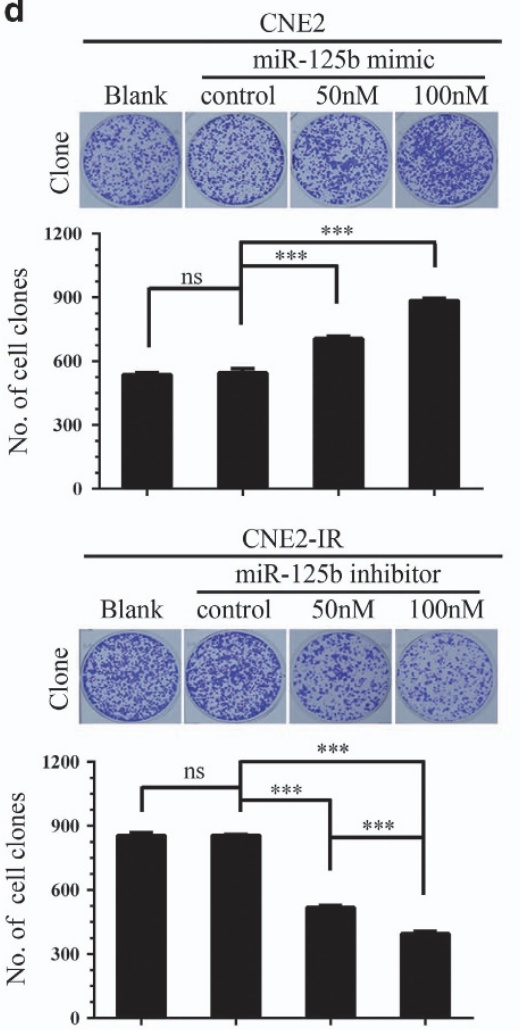

$\mathbf{f}$

antagomir control miR-125b antagomir
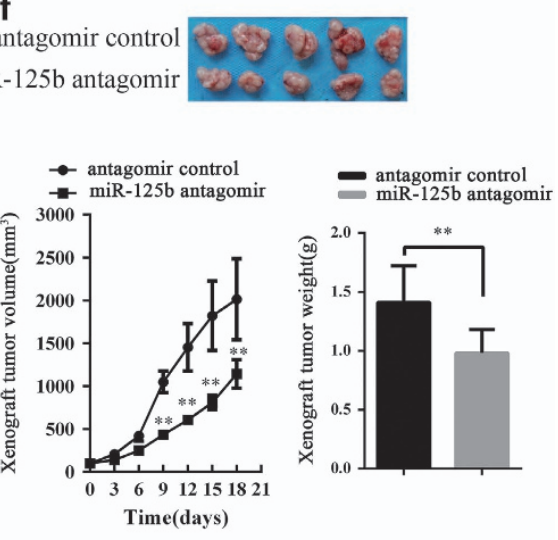

Figure 2 The effects of miR-125b on NPC cell proliferation, apoptosis, and xenograft growth. (a) QRT-PCR analysis of miR-125b expression levels in the NPC cell lines CNE2 and CNE2-IR. Analysis of cell proliferation by CCK-8 (b), EdU incorporation (c) and plate clone formation (d) assay in the miR-125b mimic-transfected CNE2 cells, miR-125b inhibitor-transfected CNE2-IR cells and their control cells. (e) Analysis of cell apoptosis by flow cytometry in the miR-125b mimic-transfected CNE2 cells, miR-125b inhibitortransfected CNE2-IR cells and their control cells. (f) MiR-125b antagomir inhibits in vivo NPC cell growth. The photography of xenograft tumors after 18 days subcutaneous implantation of control or miR-125b antagomir-injected CNE2-IR CNE2 cells (top); growth and weight of the xenograft tumors (bottom). $n=5$ mice per group. Means, S.D.s, and statistical significance are denoted; ${ }^{*} P<0.05 ;{ }^{* \star} P<0.01 ;{ }^{* * *} P<0.001$; ns, no significance 
results demonstrate that high miR-125b expression promotes NPC cell proliferation and inhibits NPC cell apoptosis in vitro.

MiR-125b antagomir inhibits in vivo NPC cell growth. To determine the effect of miR-125b on in vivo NPC cell growth, we generated subcutaneous tumors in nude mice using CNE2-IR cells. Control or miR-125b antagomir was injected into the subcutaneous tumors, and then tumor growth was assessed. As shown in Figure 2f, growth of miR-125b antagomir-injected tumors was significantly lower than that of control antagomir-injected tumors as demonstrated by tumor growth and weight, demonstrating that inhibition of miR-125b expression reduces NPC xenograft tumor growth.

MiR-125b promotes NPC cell proliferation and inhibits NPC cell apoptosis by targeting A20. To confirm A20 as a direct target of miR-125b, we co-transfected a dual luciferase reporter plasmid with wild-type A20 3'-UTR into CNE2 cells with control or miR-125b mimic. The results revealed a significant reduction in luciferase activity in miR-125b mimictransfected cells compared with control mimic-transfected cells, whereas miR-125b mimic had no obvious effects on the luciferase activity of a dual luciferase reporter plasmid without A20 $3^{\prime}$-UTR or with mutated A20 $3^{\prime}$-UTR in the miR-125bbinding site (Figure 3a). Moreover, A20 level was significantly decreased in the miR-125b mimic-transfected CNE2 cells, whereas significantly increased in the miR-125b inhibitortransfected CNE2-IR cells as compared with their respective control cells (Figure 3a). These results confirm that A20 is a direct target of miR-125b in NPC cells.

Next, we analyzed whether A20 mediates miR-125bregulating NPC cell proliferation and apoptosis. We established CNE2-IR cell lines with A20 overexpression (OE), CEN2 cell lines with A20 knockdown (KD) and their respective control cell lines (Figure $3 b$ ), and observed that A20 OE significantly decreased cell proliferation and induced cell apoptosis, whereas A20 KD significantly increased cell proliferation and inhibited cell apoptosis (Figures $3 \mathrm{c}$ and d), phenocopying those seen in the miR-125b inhibitor and mimictransfected NPC cells, respectively. Moreover, A20 KD abolished the effects of miR-125b inhibitor on the proliferation and apoptosis of CNE2-IR cells (Figures 4a and b), and A20 $\mathrm{OE}$ abolished the effects of miR-125b mimic on the proliferation and apoptosis of CNE2 cells (Figures 4c and d). Taken together, our results demonstrate that miR-125 regulates NPC cell proliferation and apoptosis by targeting A20.

A20 inhibits in vivo NPC cell growth. Tumor formation assay in nude mice was performed to determine the effects of A20 on NPC cells growth in vivo. The results showed that A20 KD significantly increased, whereas A20 OE significantly decreased the growth of NPC xenograft tumors as demonstrated by tumor growth and weight (Figures $5 \mathrm{a}$ and b). Transferase-mediated dUTP nick end labeling (TUNEL) assay showed that $A 20 \mathrm{KD}$ significantly decreased, whereas A20 OE significantly increased the number of apoptotic cells in the xenograft tumors (Figure 5c). Immunohistochemical staining showed that A20 KD significantly increased, whereas A20 OE significantly decreased the number of $\mathrm{Ki}-67$ positive cells, that is, proliferation cells in the xenograft tumors (Figure 5c). Moreover, A20 KD significantly increased, whereas A20 OE significantly decreased the expression of p-p65 (RelA) in the xenograft tumors (Figure 5c). Collectively, these results suggest that A20 inhibits in vivo NPC cells growth possibly through inhibiting cells proliferation and inducing cell apoptosis, supporting that miR-125b regulates NPC cell proliferation and apoptosis by targeting A20.

NF-кB mediates miR-125b/A20-regulating NPC cell proliferation and apoptosis. Activation of NF-KB signaling pathway has a crucial role in the development and progression of $\mathrm{NPC},{ }^{9-12}$ and $\mathrm{A} 20$ is a negative regulator of $\mathrm{NF}-\kappa \mathrm{B}$ signaling pathway. ${ }^{15-19}$ Therefore, we investigated whether NF- $K B$ mediates miR-125b/A20-regulating NPC cell proliferation and apoptosis. The results showed that either miR-125b mimic or A20 KD significantly enhanced the phosphorylated levels of $\mathrm{IKKa} / \beta, \mathrm{I}_{\kappa} \mathrm{B} a$, and $\mathrm{p} 65$, the nuclear translocation of p-p65 and NF- $K B$ luciferase reporter activity in the CNE2 cells, whereas either miR-125b inhibitor or A20 OE significantly reduced the phosphorylated levels of $\mathrm{IKK} \alpha / \beta, \mathrm{I}_{\kappa} \mathrm{B} a$, and $\mathrm{p} 65$, the nuclear translocation of $\mathrm{p}-\mathrm{p} 65$ and $\mathrm{NF}-\kappa \mathrm{B}$ luciferase reporter activity in the CNE2-IR cells (Figures 6a-d). Importantly, A20 OE abrogated activity of NF- $K \mathrm{~B}$ increased by miR-125b inhibitor in the CNE2 cells, and $A 20 \mathrm{KD}$ restored activity of NF- $K B$ decreased by miR-125b mimic in the CNE2IR cells (Figures $7 a-c)$. Collectively, these results demonstrate that miR-125b regulates the activity of $N F-K B$ signaling pathway by targeting A20.

Next, we determined whether NF-kB mediates miR-125b/ A20-regulating NPC cell proliferation and apoptosis. We observed that either exogenous $I_{K B} a$ OE or NF- $\kappa B$ inhibitor BAY11-7082 treatment significantly abolished the effects of A20 KD on NPC cell proliferation and apoptosis, whereas exogenous p65 OE restored the effects of A20 OE on NPC cell proliferation and apoptosis (Figures $8 \mathrm{a}-\mathrm{c}$ ). These results demonstrate that NF-KB signaling mediates miR-125b/A20regulating NPC cell proliferation and apoptosis.

Levels of miR-125b, A20, and p-p65 are correlated in human NPC biopsies. As our data demonstrate that miR-125b regulates NPC cell proliferation and apoptosis by targeting $\mathrm{A} 20 / \mathrm{NF}-\mathrm{kB}$, we next determined whether the levels of miR-125b, A20 and p-p65 were correlated in human NPC biopsies. Our immunohistochemistry showed that A20 expression was significantly lower, whereas p-p65 was significantly higher in the NPC tissues than that in the NNM (Figure 1c, Table 1). Correlation analyses revealed that miR-125b level was negatively associated with A20 level ( $r=-0.61, P<0.001$ ), whereas positively associated with p-p65 level ( $r=0.45, P<0.001)$, and A20 level was negatively associated with p-p65 level $(r=-0.38, P<0.001)$. Together, these results indicate that high $\mathrm{miR}-125 \mathrm{~b}$ expression appears to be associated with downregulation of $\mathrm{A} 20$ and the activations of NF- $\kappa \mathrm{B}$ in the NPC tissues, and these misregulations might contribute to NPC development.

\section{Discussion}

The dysregulation of miRNA has been implicated in human cancers. ${ }^{2,3}$ In this study, we focused on miR-125b, because 
the role and mechanism of miR-125b in NPC are unclear. We observed that miR-125b was frequently upregulated in the NPC tissues relative to NNM, and its increment correlated with poor patient survival, outlining a potential marker for predicting the prognosis of NPC patients. To gain insight into miR-125b function in the pathogenesis of NPC, we analyzed the effects of miR-125b on NPC cell proliferation and apoptosis, and found that downregualtion of miR-125b decreased cell

a
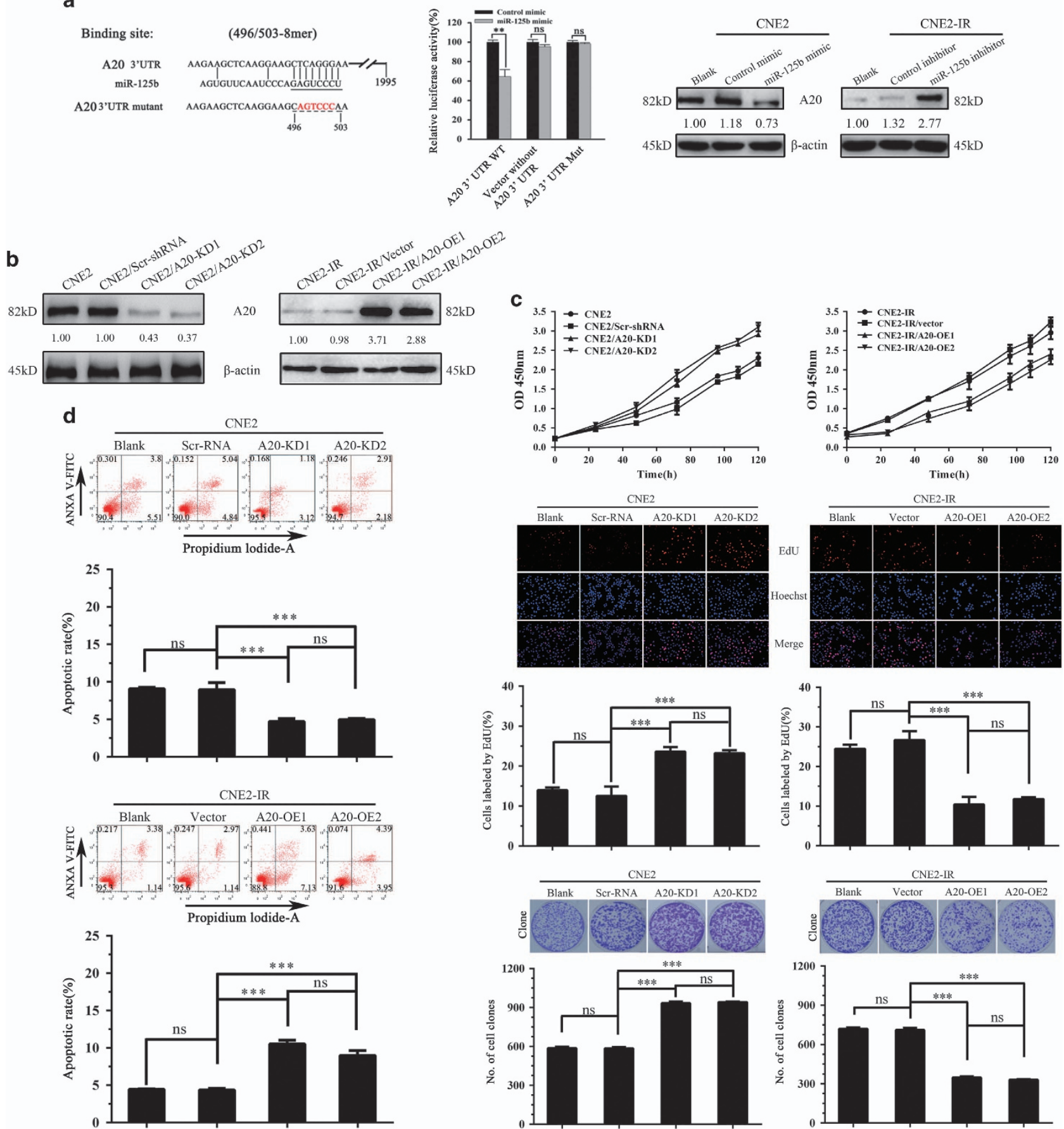

Figure 3 Target A20 of miR-125b regulates NPC cell proliferation and apoptosis. (a) $3^{\prime}$-UTR dual luciferase reporter assay showing A20 as a direct target of miR-125b in NPC cells. (left) The predicted miR-125b binding sites in the 3'-UTR of wild-type (wt) A20 and mutant (mt) A20 3'-UTR; (middle) Luciferase activity of wt and mt A20 3'-UTR and without A20 3'-UTR dual luciferase reporter vector in the CNE2 cells transfected with control or miR-125b mimic; (right) Western blot analysis showing A20 levels in the miR-125b mimic-transfected CNE2, miR-125 inhibitor-transfected CNE2-IR cells and their respective control cells. (b) Western blot analysis showing A20 levels in the A20 KD CNE2 cells, A20 OE CNE2-IR cells and their respective control cells. (c) Analysis of cell proliferation by CCK-8 (top), EdU incorporation (middle) and plate clone formation (bottom) assay in A20 KD CNE2 cells, A20 OE CNE2-IR cells and their respective control cells. (d) Analysis of cell apoptosis by flow cytometry in the A20 KD CNE2 cells, A20 OE CNE2-IR cells and their respective control cells. Three experiments were done; means, S.D.s, and statistical significance are denoted; ${ }^{* \star} P<0.01 ;{ }^{* \star *} P<0.001 ;$ ns, no significance. Vector, transfected with an empty vector; $\mathrm{KD}$, knockdown; $\mathrm{OE}$, overexpression 
proliferation and induced cell apoptosis, whereas upregualtion of miR-125b increased cell proliferation and inhibited cell apoptosis, suggesting that miR-125b acts as oncogene in NPC.

a
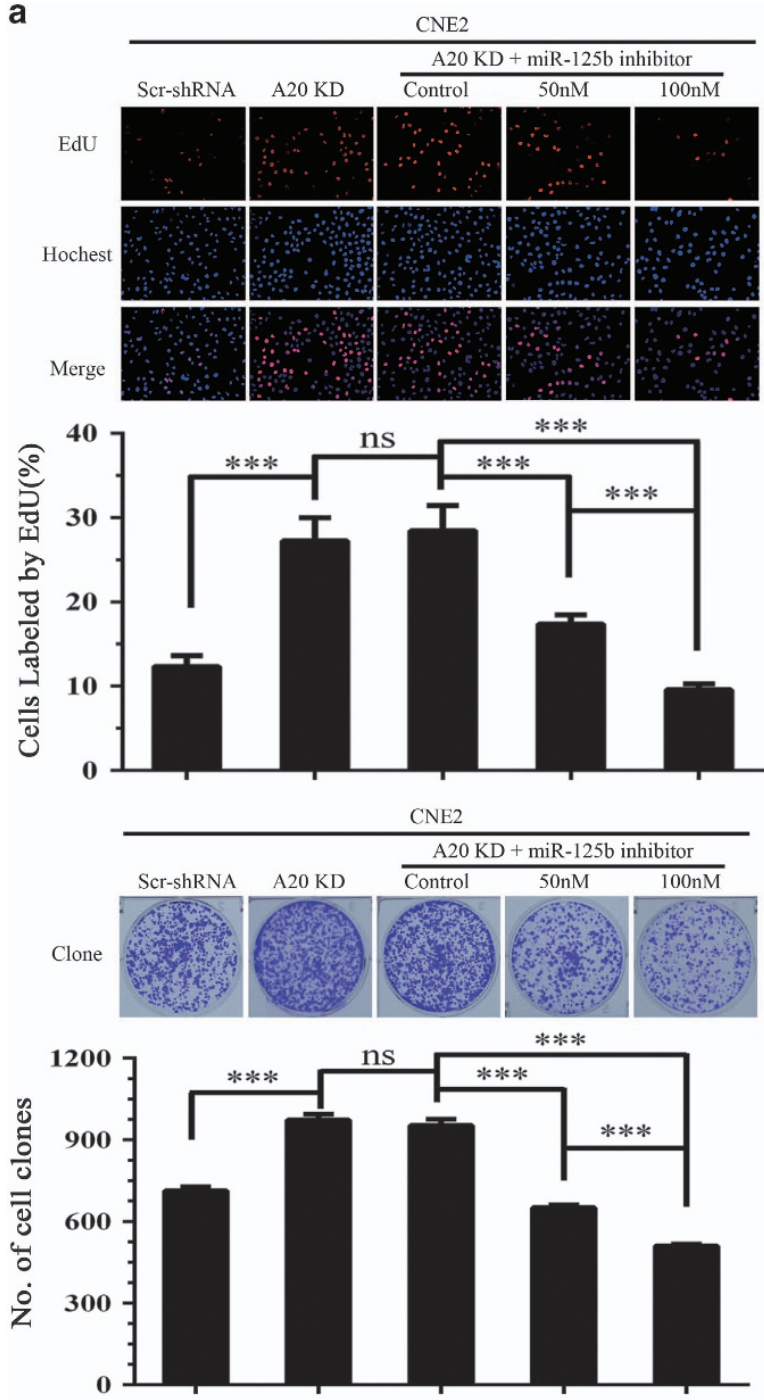

b

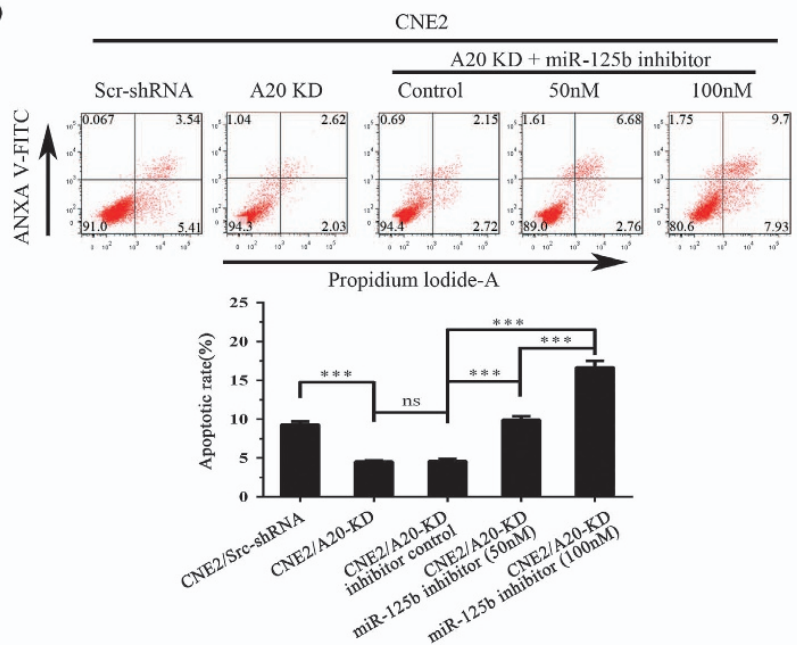

As miRNAs exert their roles through inhibiting target mRNA translation, thus identification of miR-125b target genes is a key step for understanding the mechanism of miR-125bregulating NPC cell proliferation and apoptosis. It has been

c
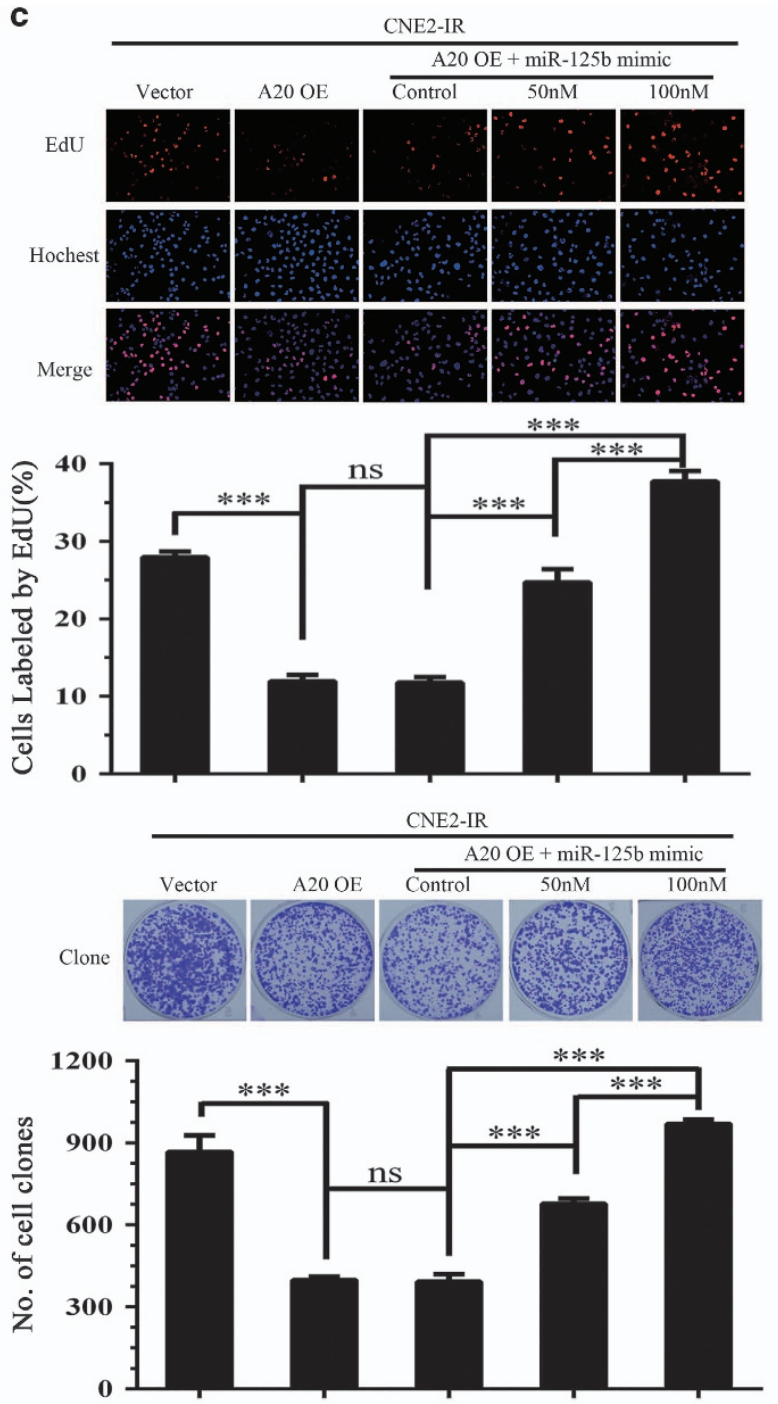

d

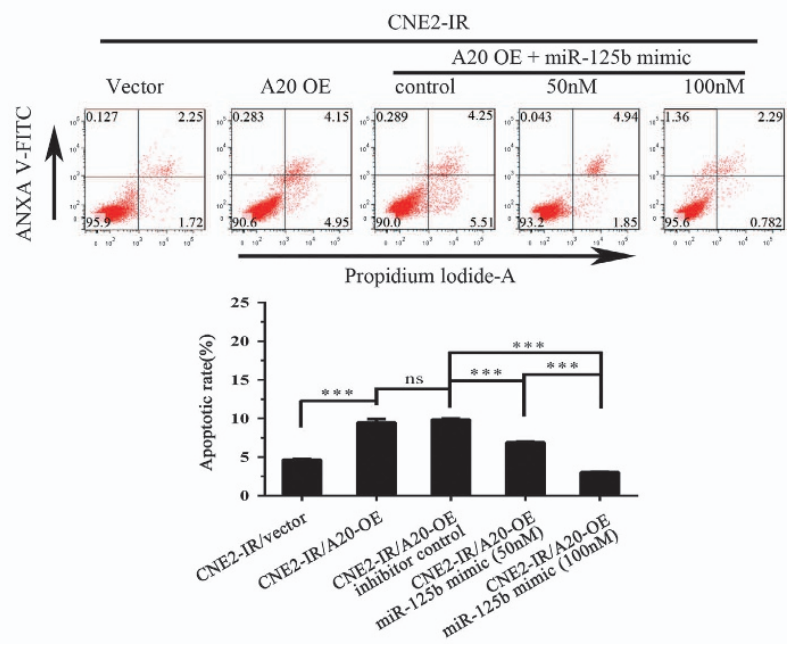


reported that miR-125b activates NF- $\kappa \mathrm{B}$ pathway by targeting $\mathrm{A} 20,{ }^{20-22}$ and activation of NF-KB signaling confers the advantages of tumor cell proliferation and survival. ${ }^{8}$ Therefore, we analyzed whether miR-125b regulates NPC cell proliferation and apoptosis by targeting A20. Our results confirm that A20 is a direct target of miR-125b in NPC cells, and miR-125b regulates NPC cell proliferation and apoptosis by targeting A20.

Numerous studies have revealed that A20 negatively regulates NF- $k B$ signaling pathway by inhibiting the function of several NF- $\kappa$ B upstream signaling transducers. ${ }^{15-20}$ Activation of $\mathrm{NF}-\kappa \mathrm{B}$ signaling pathway has a crucial role in the development and progression of NPC. ${ }^{9-12}$ Therefore, we investigated whether A20 mediates miR-125b-regulating NPC cell proliferation and apoptosis by activating NF- $k \mathrm{~B}$. We observed that miR-125b mimic or A20 KD enhanced, whereas
miR-125b inhibitor or A20 overexpression reduced the activity of NF- $k$ B signaling pathway in NPC cells. Importantly, A20 overexpression abrogated activation of $N F-K B$ signaling induced by miR-125b mimic, and A20 KD restored activity of NF- $K B$ signaling decreased by miR-125b inhibitor in the NPC cells. These results demonstrate that miR-125b activates NF- $\kappa \mathrm{B}$ by targeting A20. We further showed that either $I_{\kappa} \mathrm{Ba}$ overexpression or BAY11-7082 abolished the effects of A20 KD on NPC proliferation and apoptosis, whereas p65 (RelA) overexpression abolished the effects of A20 overexpression on NPC cell proliferation and apoptosis. In the clinical NPC samples, p-p65 (RelA) level was negatively associated with A20 level, whereas positively associated with miR-125b level. Taken together, our results demonstrate that NF-kB signaling mediates miR-125b/A20-regulating NPC cell proliferation and apoptosis. a

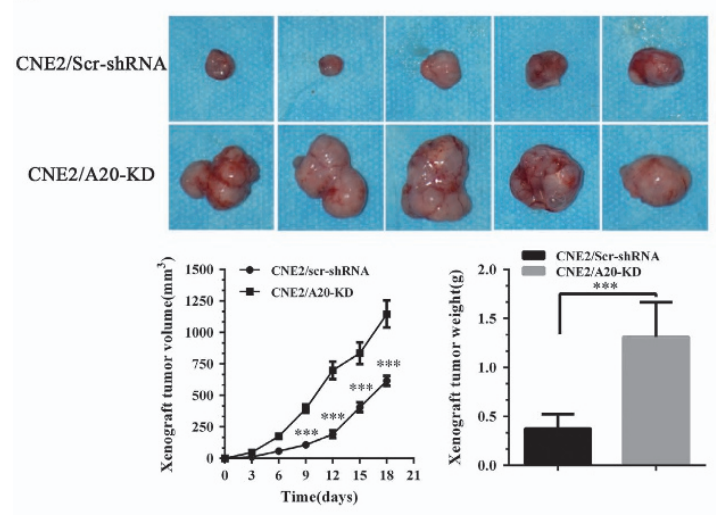

b
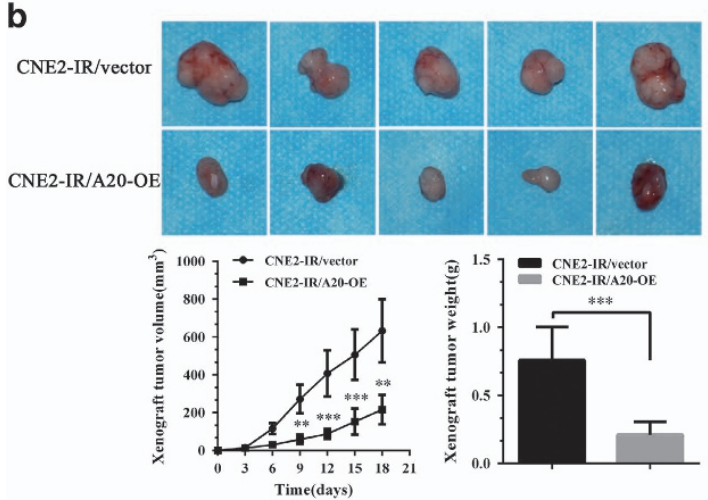

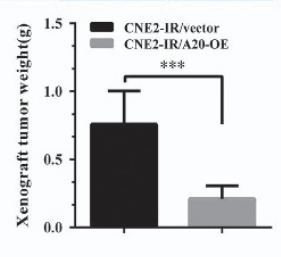

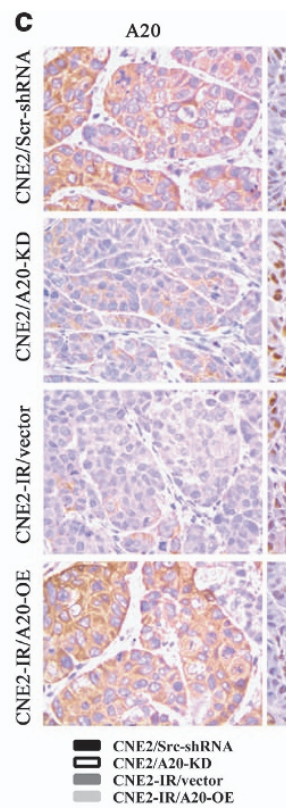

A20

p-p65

TUNEL

Ki-67
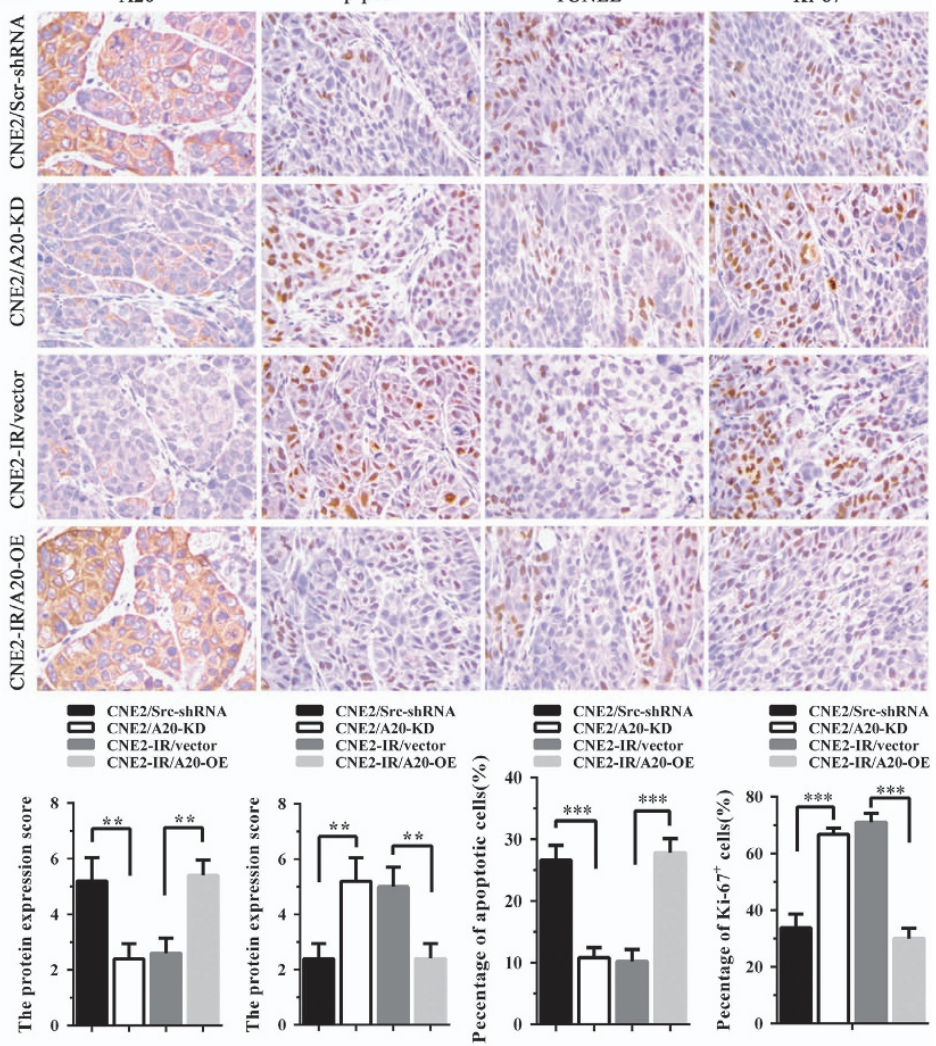

Figure 5 A20 inhibits in vivo NPC cell growth. (a) The representative photography of xenograft tumors after 18 days subcutaneous implantation of A20 KD CNE2 cells and control cells (top); Growth and weight of xenograft tumors generated by A20 KD CNE2 cells and control cells (bottom). (b) The representative photography of xenograft tumors after 18 days subcutaneous implantation of A20 OE CNE2-IR cells and control cells (top); Growth and weight of xenograft tumors generated by A20 OE CNE2-IR cells and control cells (bottom). (c) Representative results of A20, p-p65 (RelA), TUNEL, and Ki-67 immunohistochemical staining (top) and statistical analysis (bottom) of xenograft tumors generated by A20 KD CNE2 cells, A20 OE CNE2-IR cells and their respective control cells. $n=10$ mice per group. Original magnification, $\times 400$. Means, S.D.s $(n=10)$, and statistical significance are denoted; ${ }^{* *} P<0.01 ;{ }^{* \star *} P<0.001$. Vector, transfected with an empty vector; $\mathrm{KD}$, knockdown; OE, overexpression

Figure 4 MiR-125b promotes NPC cell proliferation and inhibits NPC cell apoptosis by targeting A20. (a) Analysis of cell proliferation by EdU incorporation (top) and plate clone formation (bottom) assay in the A20 KD CNE2 cells transfected with miR-125b inhibitor and control cells. (b) Analysis of cell apoptosis by flow cytometry in the A20 KD CNE2 cells transfected with miR-125b inhibitor and control cells. (c) Analysis of cell proliferation by EdU incorporation (top) and plate clone formation (bottom) assay in the A20 OE CNE2-IR cells transfected with miR-125b mimic and control cells. (d) Analysis of cell apoptosis by flow cytometry in the A20 OE CNE2-IR cells transfected with miR-125b mimic and control cells. Three experiments were done; Means, S.D.s, and statistical significance are denoted; ${ }^{* \star *} P<0.001 ;$ ns, no significance. Vector, transfected with an empty vector; $\mathrm{KD}$, knockdown; OE, overexpression 
a

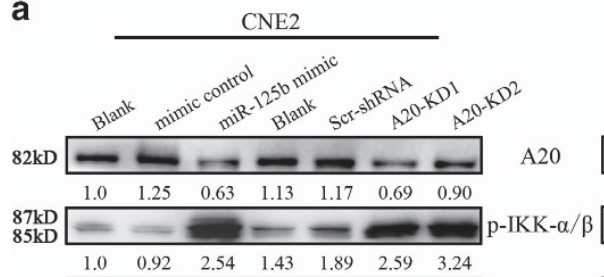

\begin{tabular}{|ccccccccc}
1.0 & 0.92 & 2.54 & 1.43 & 1.89 & 2.59 & 3.24 \\
\hline 85kD & & & - & - & - & & & \\
\hline 1.0 & 1.07 & 0.84 & 0.90 & 1.01 & 0.97 & 1.09 \\
\hline & IKK- $\alpha$ & \\
\hline 1.0 & 1.61 & 2.79 & 1.29 & 1.61 & 2.86 & 2.71 \\
\hline
\end{tabular}

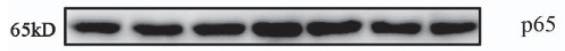

\begin{tabular}{lllllll|}
\hline 1.0 & 1.07 & 1.17 & 1.41 & 1.20 & 1.13 & 1.16
\end{tabular}

$40 \mathrm{kD}=-\mathrm{m}-\mathrm{m}-\mathrm{m}-\mathrm{I \kappa B}-\alpha$

\begin{tabular}{lllllll|}
1.0 & 1.91 & 2.57 & 1.37 & 1.37 & 2.29 & 2.37
\end{tabular}

$39 \mathrm{kD}=\mathrm{I \kappa B}-\alpha$

$\begin{array}{lllllll}1.0 & 1.07 & 0.58 & 0.81 & 0.70 & 0.49 & 0.48\end{array}$

$52 \mathrm{kD} \longrightarrow$

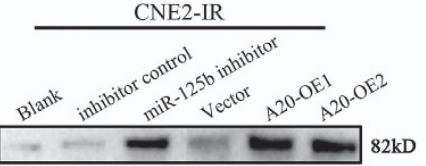

\begin{tabular}{llllll}
\hline 1.0 & 1.56 & 5.01 & 3.60 & 6.06 & 5.88
\end{tabular}

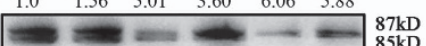

\begin{tabular}{llllll}
1.0 & 1.02 & 0.57 & 0.91 & 0.38 & 0.73 \\
\hline & & & & &
\end{tabular}

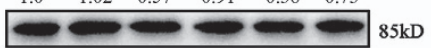

$\begin{array}{llllll}1.0 & 0.92 & 0.92 & 1.12 & 0.95 & 0.89\end{array}$

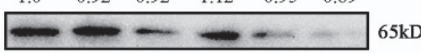

$\begin{array}{llllll}1.0 & 1.07 & 0.72 & 0.90 & 0.64 & 0.45\end{array}$
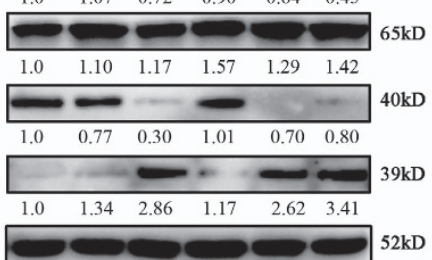

C

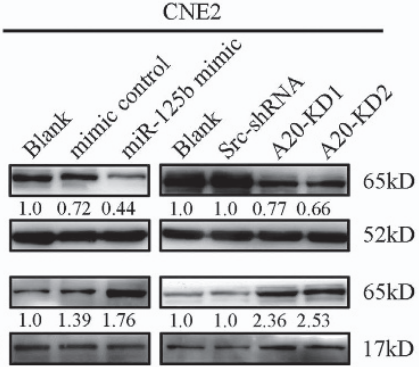

CNE2-IR

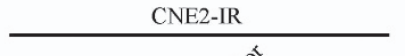

势 $\begin{gathered}\mathrm{p} 65 \\ \text { Tubulin }\end{gathered}$

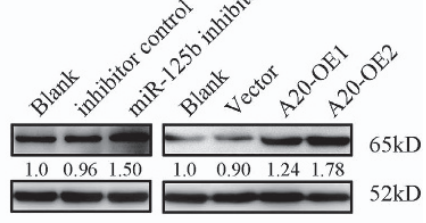

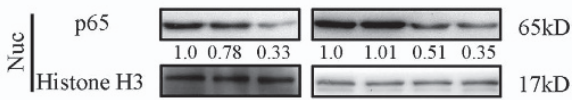

b

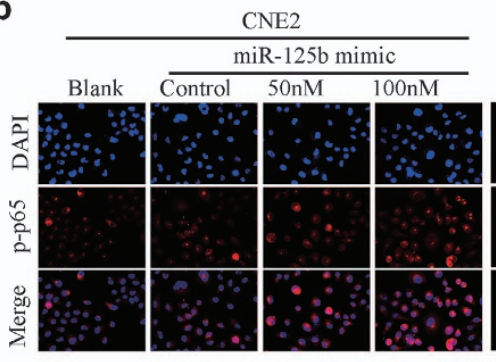

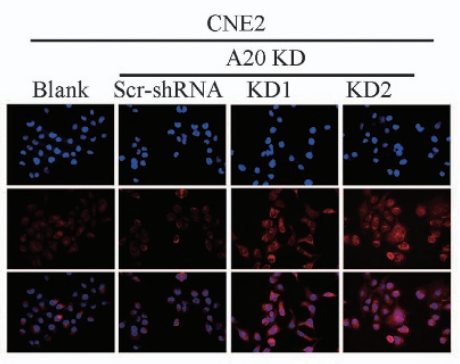

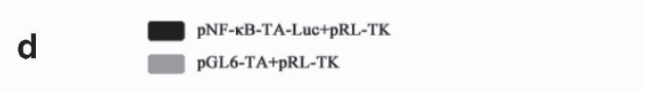

趂

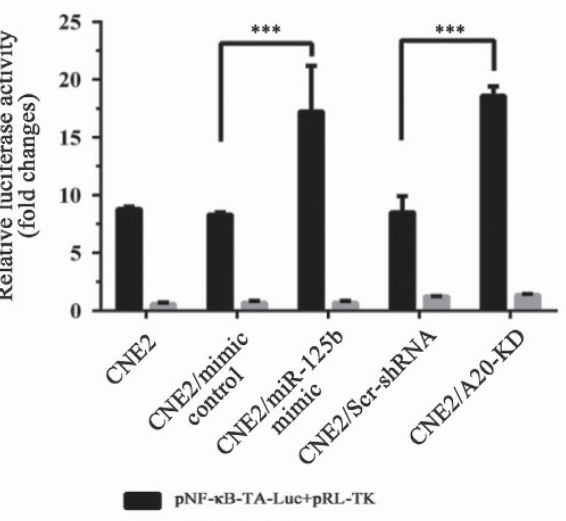

pGL6-TA+pRL-TK

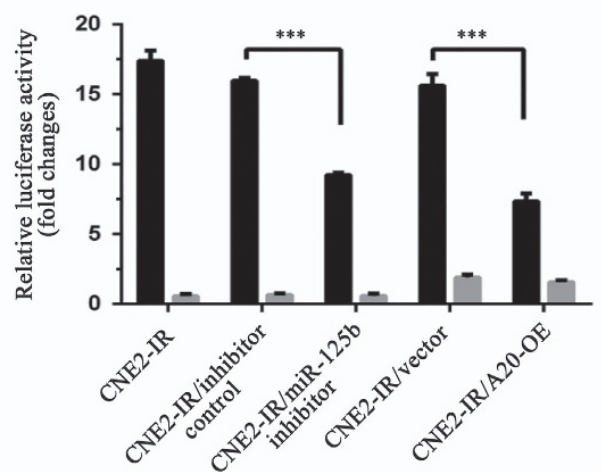

Figure 6 MiR-125b regulates the activity of NF- $k B$ signaling pathway by targeting A20 in NPC cells. (a) Western blot analysis showing the levels of $p-\mid K K \alpha / \beta, p-1 / B \alpha, p-p 65$ (RelA), IKK $\alpha / \beta, I_{K} B \alpha$, and p65 in the miR-125b mimic-transfected CNE2 cells, miR-125 inhibitor-transfected CNE2-IR cells, A20 KD CNE2 cells, A20 OE CNE2-IR cells, and their respective control cells. (b) Representative immunofluorescent staining showing the nuclear translocation of p-p65(RelA) in the miR-125b mimic-transfected CNE2 cells, miR-125 inhibitor-transfected CNE2-IR cells, A20 KD CNE2 cells, A20 OE CNE2-IR cells, and their respective control cells. (c) Western blot analysis showing p65(RelA) expression in the nuclear and cytoplasmic fractions of miR-125b mimic-transfected CNE2 cells, miR-125 inhibitor-transfected CNE2-IR cells, A20 KD CNE2 cells, A20 OE CNE2-IR cells, and their respective control cells. Nuc, nuclear fraction; Cyt, cytoplasmic fraction. (d) A luciferase reporter assay showing p65 transcriptional activity in the miR-125b mimic-transfected CNE2 cells, miR-125 inhibitor-transfected CNE2-IR cells, A20 KD CNE2 cells, A20 OE CNE2-IR cells, and their respective control cells. Three experiments were done; Means, S.D.s and statistical significance are denoted; ${ }^{* \star \star} P<0.001$; ns, no significance. Vector, transfected with an empty vector; KD, knockdown; OE, overexpression 
Numerous studies have indicated that A20 is involved in the pathogenesis of various types of lymphoid malignancies, ${ }^{24-28}$ multiple myeloma ${ }^{29,30}$ and non-small cell lung cancer ${ }^{31}$ duo to its genetic or epigenetic inactivation, leading to A20 downregulation, which functions as a tumor suppressor. It is also reported that $\mathrm{A} 20$ acts as oncogene in glioblastoma, ${ }^{32,33}$

a

Scr-shRNA

A20-KD

miR-125b inhibitor control

miR-125b inhibitor 50nM

miR-125b inhibitor $100 \mathrm{nM}$

A20

\begin{tabular}{ccccc}
\multicolumn{5}{c}{ CNE2 } \\
\hline- & + & - & - & - \\
- & - & + & + & + \\
- & - & + & - & - \\
- & - & - & + & - \\
- & - & - & - & +
\end{tabular}

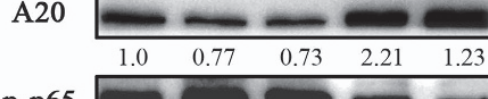

$82 \mathrm{kD}$

p-p65

$65 \mathrm{kD}$

p65

$\begin{array}{lllll}1.0 & 1.18 & 1.14 & 0.79 & 0.57\end{array}$

$65 \mathrm{kD}$

Tubulin

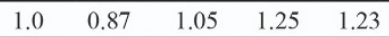

$\begin{array}{lllll}1.0 & 0.87 & 1.05 & 1.25 & 1.23\end{array}$

$52 \mathrm{kD}$

Vector

A20-OE

miR-125b mimic control

miR-125b mimic 50nM

miR-125b mimic $100 \mathrm{nM}$

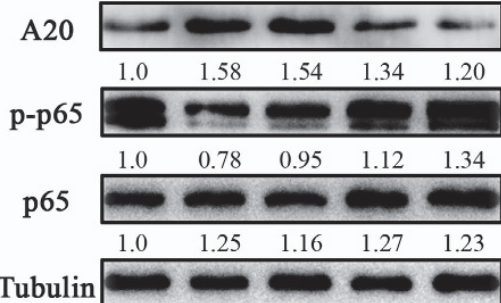

$82 \mathrm{kD}$

CNE2-IR

\begin{tabular}{lllll}
\hline- & + & - & - & - \\
- & - & + & + & + \\
- & - & + & - & - \\
- & - & - & + & - \\
- & - & - & - & +
\end{tabular}

$65 \mathrm{kD}$

$65 \mathrm{kD}$

Tubulin

$52 \mathrm{kD}$
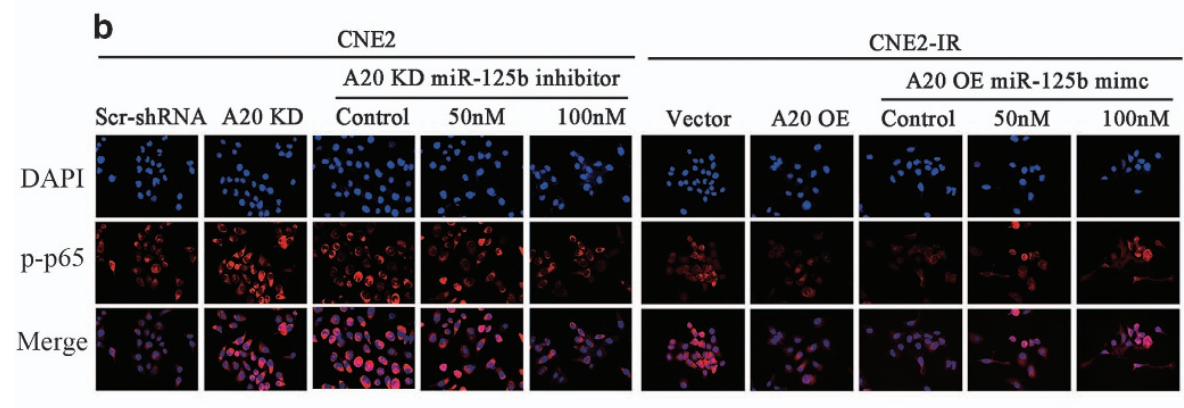

C
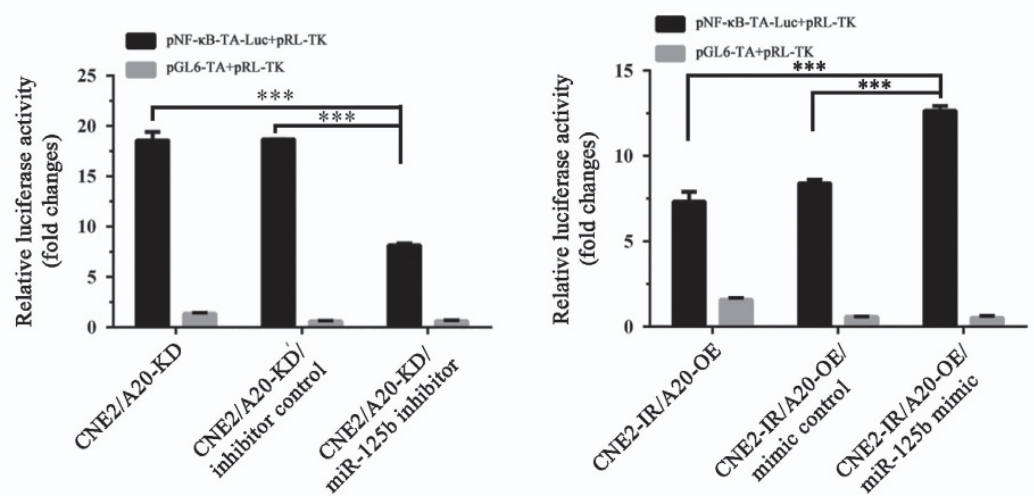

Figure 7 The effect of A20 expression changes on miR-125b-regulating NF- $k B$ activity. Western blot analysis showing $p-p 65($ RelA) levels (a), representative immunofluorescent staining showing the nuclear translocation of p-p65 (b), and luciferase reporter assay showing p65 transcriptional activity (c) in the A20 KD CNE2 cells transfected with miR-125b inhibitor, A20 OE CNE2-IR cells transfected with miR-125b mimic, and their respective control cells. Three experiments were done; Means, S.D.s and statistical significance are denoted; ${ }^{* \star} P<0.001$. Vector, transfected with an empty vector; $\mathrm{KD}$, knockdown; OE, overexpression 
hepatitis $\mathrm{B}$ virus-related hepatocellular carcinoma, ${ }^{34}$ and gastric cancer. ${ }^{35}$ However, the expression and function of A20 in NPC are poorly understood. In this study, we observed a
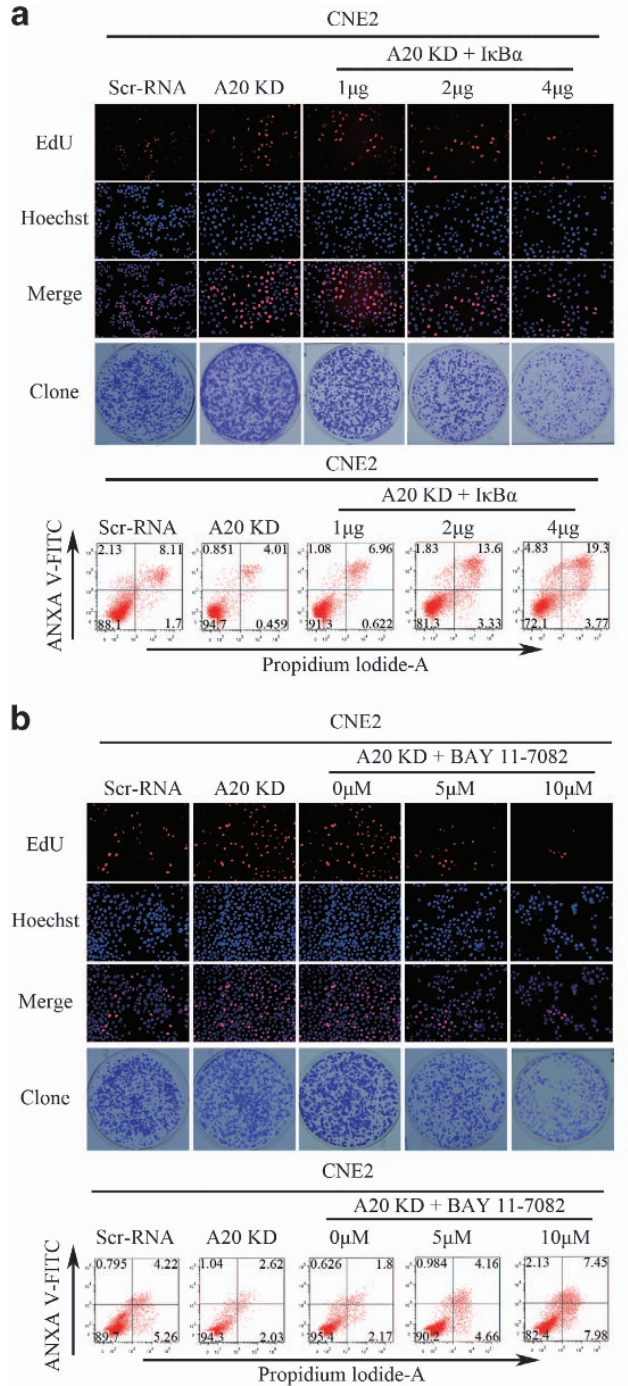

C

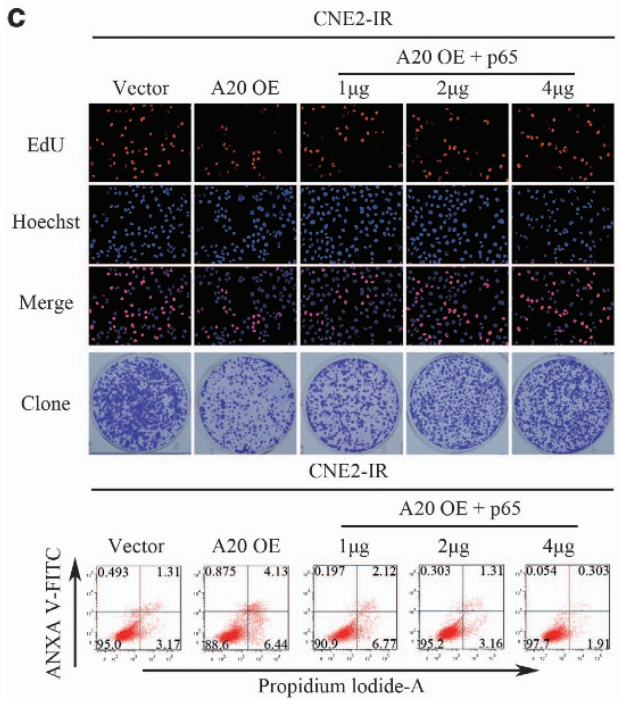

that $A 20$ was significantly decreased in NPC tissues relative to NNM, inhibited NPC cell proliferation, and induced NPC apoptosis; A20 KD significantly increased while A20
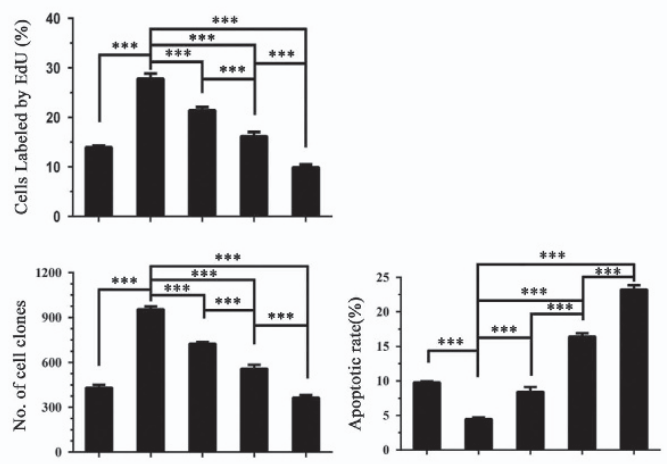

Scr-RNA

A20-KD

IкB $\alpha 1 \mu$

I $\mathrm{B} \alpha 2 \mu \mathrm{g}$
I $\mathrm{B} \alpha 4 \mu \mathrm{g}$
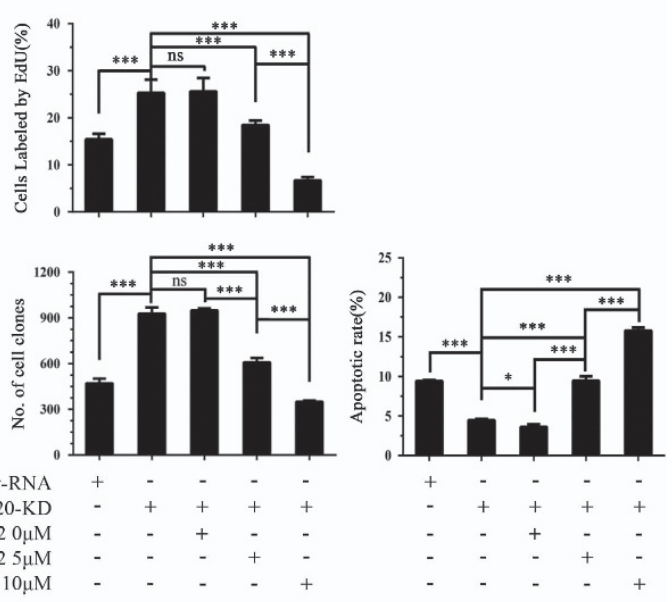

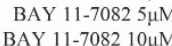
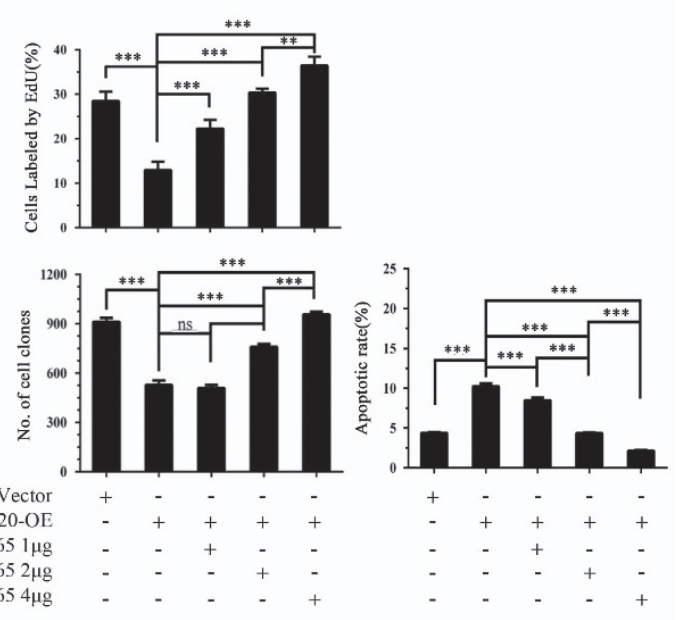
ovexpression significantly decreased the growth of NPC xenograft tumors in nude mice possibly through inhibiting cell proliferation and inducing cell apoptosis. The results strongly suggest that A20 functions as a tumor suppressor in NPC.

Although A20/NF- $K B$ signaling axis seems to largely account for the phenotype of NPC cells induced by miR-125b, indeed a single miRNA can target multiple mRNAs to regulate gene expression. ${ }^{36}$ Therefore, there might be other molecules such as BAK1, PPP1CA, and $p 53,{ }^{37-41}$ which are also targeted by miR-125b in NPC cells. We also observed that miR-125b negatively modulated p53 expression in NPC cells, but it still promoted cell proliferation and inhibited cell apoptosis in p53 KD NPC CNE2 cell line that was established previously by us (Supplementary Figure 1). ${ }^{42}$ The results suggest that miR-125b promotes NPC cell proliferation and inhibits NPC cell apoptosis by p53-independent manner. ${ }^{43}$

In summary, our data demonstrate that miR-125b is frequently upregulated in the NPC biopsies, and is an independent predictor for NPC patient survival; miR-125b regulates NPC cell proliferation and apoptosis by targeting A20/NF- $\kappa$ B signaling pathway; A20 inhibits NPC cell proliferation and induces NPC cell apoptosis both in vitro and growth in vivo. Our data demonstrate that miR-125b act as an oncogene, whereas A20 act as a tumor suppressor in NPC, and suggests that targeting $\mathrm{miR}-125 \mathrm{~b} / \mathrm{A} 20 / \mathrm{NF}-k \mathrm{~B}$ signaling axis is a promising approach for NPC patients with high miR-125b expression.

\section{Materials and Methods}

Patients and tissue samples. A total of 111 NPC patients without distant metastasis (MO stage) at the time of diagnosis who were treated by radical radiotherapy alone in the Affiliated Cancer Hospital of Central South University, China between January 2006 and December 2008 were recruited in this study. The radiotherapy was administered for a total dose of $60-70$ Gy (2 Gy/fraction, 5 days a week). The neck received 60Gy for lymph node-negative cases and 70 Gy for lymph node-positive cases. NPC tissue biopsies were obtained at the time of diagnosis before any therapy, fixed in $4 \%$ formalin and embedded in paraffin. We also acquired 30 cases of formalin-fixed and paraffin-embedded NNM in the same period. On the basis of the 1978 WHO classification, ${ }^{44}$ all tumors were

Table 1 The expression levels of A20 and phospho-p65 in the NNM and NPC tissues

\begin{tabular}{lccc}
\hline & NNM ( $\boldsymbol{n}, \%)$ & NPC $(\boldsymbol{n}, \%)$ & $\boldsymbol{P}\left(\boldsymbol{x}^{2}\right.$-test $)$ \\
\hline A20 & & & \\
Low (0-3) & $7(23.33)$ & $68(61.26)$ & $<0.001$ \\
High (4-6) & $23(76.67)$ & $43(38.74)$ & \\
& & & \\
p-p65 & & & \\
Low (0-3) & $28(93.33)$ & $36(32.43)$ & $<0.001$ \\
High (4-6) & $2(6.67)$ & $75(67.57)$ & \\
\hline
\end{tabular}

Abbreviations: NNM, normal nasopharyngeal mucosa; NPC, nasopharyngeal carcinoma. histopathologically diagnosed as poorly differentiated squamous cell carcinomas (WHO type III). The clinical stage of the patients was classified according to the 2008 NPC staging system of China. ${ }^{45}$

The patients were followed up, and the follow-up period at the time of analysis was $>72$ months (average, $77.5 \pm 11.8$ months). DFS was calculated as the time from the completion of primary radiotherapy to the date of pathological diagnosis or clinical evidence of local failure and/or distant metastasis. OS was defined as the time from the initiation of primary radiotherapy to the date of cancer-related death or when censured at the latest date if patients were still alive. The clinicopathologic parameters of the patients used in the present study are shown in Supplementary Table S2.

Cell lines. Human NPC cell lines CNE2-IR and CNE2 cells have been described previously by us, ${ }^{23}$ and cultured with RPMl-1640 medium supplemented with $10 \%$ fetal bovine serum (Life Technologies, Carlsbad, CA, USA) at $37{ }^{\circ} \mathrm{C}$ in $5 \% \mathrm{CO}_{2}$. The cell lines were routinely tested for presence of mycoplasma with 4,6-diamidino-2phenylindole staining, and were mycoplasma free.

QRT-PCR. QRT-PCR was performed as described previously by us. ${ }^{23}$ In brief, total RNA was extracted from NPC cells with Trizol reagent (Invitrogen, Carlsbad, CA, USA), or from paraffin-embedded NPC and NNM with RecoverAll total nucleic acid isolation kit (Ambion, Austin, TX, USA) according to the manufacturer's instructions. For miR-125b qRT-PCR, $2 \mu \mathrm{g}$ of total RNA was reversely transcribed for cDNA using a reverse transcription (RT) kit according to the manufacturer's instructions (Promega, Madison, WI, USA) and miR-125b specific primer (BulgeLoop miRNA qPCR primer). The RT products were amplified by real-time PCR using miScript SYBR green PCR kit (Qiagen, Hilden, Germany) according to the manufacturer's instructions. For A20 mRNA qRT-PCR, $2 \mu \mathrm{g}$ of total RNA was reversely transcribed for CDNA using a RT kit according to the manufacturer's protocol and Oligo dT primer (Promega) according to the manufacturer's instructions. The RT products were amplified by real-time PCR using QuantiFast SYBR green PCR kit (Qiagen) according to the manufacturer's instructions. The products were quantitated using $2^{-D D C t}$ method against U6 or GAPDH for normalization. The primer sequences were synthesized by RiboBio (Guangzhou, China) and summarized in Supplementary Table S3.

Luciferase activity assay. For the A20 $3^{\prime}$-UTR luciferase reporter assay, a dual luciferase reporter plasmid with wild-type A20 3'-UTR (HmiT018145-MT01, GeneCopoeia), without A20 3'-UTR (CmiT000001-MT01, GeneCopoeia), or with mutated A20 $3^{\prime}$-UTR in the predicted miR-125b binding site constructed by GeneCopoeia, and miR-125b or control mimic (RiboBio) were co-transfected into NPC cells using Lipofectamine 2000 (Invitrogen) according to the manufacturer's instructions. For the NF-kB luciferase reporter assay, a reporter plasmid containing human NF-kB/p65 response element (pNF-kB-TA-luc) (Beyotime, Nanjin, China) or pGL6-TA plasmid without NF-kB response element (Beyotime) and pRL-TK plasmid (Promega) were co-transfected into the indicated NPC cells using Lipofectamine 2000. Cells were harvested $48 \mathrm{~h}$ after transfection, both firefly luciferase and renilla luciferase activities were measured using the dual luciferase reporter assay system (Promega) according to the manufacturer's instructions, and luciferase activity was estimated using a luminometer (Promega).

Transfection of miR-125b mimic and inhibitor into cells. A total of 50 and $100 \mathrm{nmol} / \mathrm{ml}$ miR-125b mimic, miR-125b inhibitor and their respective negative control (Ribobio) were transfected into the indicated NPC cells using RiboFect CP transfection kit (Ribobio) according to the manufacturer's instructions, respectively. Twenty-four hours after transfection, cells were subjected to further analysis.

Establishment of NPC cell lines with A20 OE and KD. Lentiviral GV248-A20 shRNA and GV248-scramble non-target shRNA vector, which was established by Genechem (Shanghai, China), and confirmed by sequencing. The

Figure 8 NF-kB mediates miR-125b/A20-regulating NPC cell proliferation and apoptosis. (a) Representative results (left) and statistical analyses (right) of EdU incorporation assay, plate clone formation assay and detection of cell apoptosis by flow cytometry in the A20 KD CNE2 cells transfected with plasmid expressing IKB $\alpha$ and control cells. (b) Representative results (left) and statistical analyses (right) of EdU incorporation assay, plate clone formation assay and detection of cell apoptosis by flow cytometry in the A20 KD CNE2 cells treated with BAY11-7082 and control cells. (c) Representative results (left) and statistical analyses (right) of EdU incorporation assay, plate clone formation assay and detection of cell apoptosis by flow cytometry in the A20 OE CNE2-IR cells transfected with plasmid expressing p65 and control cells. Three experiments were done; Means, S.D.s, and statistical significance are denoted; ${ }^{*} P<0.05$; ${ }^{* \star *} P<0.001$; ns, no significance. Vector, transfected with an empty vector; KD, knockdown; OE, overexpression 
target for human A20 shRNA was 5'-CACTGGAAGAAATACACATAT-3', the KD efficiency of which has been validated. ${ }^{33}$ A20 expression plasmid (EX-K6040) and control vector pReceiver-M13 were purchased from GeneCopoeia. Cells were infected or transfected with the lentiviral vectors or plasmids according to the manufacturer's instructions, and then selected using neomycin or puromycin for 2 weeks. NPC cell lines with stable KD or OE of A20 and control cell lines were obtained.

CCK-8 assay. Cell proliferation was measured using a CCK- 8 kit. In brief, the cells were plated at $1 \times 10^{3}$ cells/well in 96-well tissue culture plates, and grew for 7 days. Every $24 \mathrm{~h}, 10 \mu \mathrm{l}$ CCK-8 reagent (Beyotime) was added to every well, and incubated for $4 \mathrm{~h}$. The absorbance of each well was read with a Bio-Tek Instruments EL310 Microplate Autoreader at $450 \mathrm{~nm}$. CCK-8 assay was performed three times in triplicate.

EdU incorporation assay. Cell proliferation was measured using EdU assay. In brief, the cells were cultured in chamber slides (Millipore, Billerica, MA, USA; $2 \times 10^{4}$ cells/well). Forty-eight hours after culture, the cells were treated with $50 \mu \mathrm{M}$ EdU (RiboBio) for an additional $2 \mathrm{~h}$ at $37^{\circ} \mathrm{C}$, and then were fixed with $4 \%$ formaldehyde for $30 \mathrm{~min}$, followed by addition of $200 \mu \mathrm{l}$ glycine $(2 \mathrm{mg} / \mathrm{ml}$; Amresco, $\mathrm{OH}, \mathrm{USA}$ ). After $5 \mathrm{~min}$, the cells were incubated with $0.5 \%$ Triton X-100 (SigmaAldrich, St. Louis, MO, USA) for $10 \mathrm{~min}$ at room temperature. Following washing with PBS for $5 \mathrm{~min}, 1 \times$ Apollo reaction reagent (RiboBio) was added and incubated at room temperature in the dark for $30 \mathrm{~min}$, and then the cells were stained with $200 \mu \mathrm{l}$ Hoechst 33342 (5 $\mu \mathrm{g} / \mathrm{ml}$; Sigma-Aldrich) for an additional $30 \mathrm{~min}$ in the dark. Cells labeled and unlabeled by EdU were counted under a Leica DMI4000 microscope, and pictures were taken. The assay was performed three times in triplicate.

Plate colony formation assay. Plate colony formation assay was performed to detect cell proliferation. In brief, $1 \times 10^{3}$ cells were plated into each well of six-well plate, and were growing for 14 days. At the end of the experiment, cell colonies were fixed with $4 \%$ paraformaldehyde, and stained with $0.5 \%$ crystal violet (Sigma-Aldrich) stained. The numbers of colony were counted under a Leica DMI4000 microscope, and pictures were taken. The assay was performed three times in triplicate.

Analysis of cell apoptosis by flow cytometry. Cell apoptosis was assessed by Annexin V-fluorescein isothiocyanate apoptosis detection kit I (BD Biosciences, San Diego, CA, USA) according to the manufacturer's instructions. In brief, $5 \times 10^{5}$ cells were collected by centrifugation, resuspended in $500 \mu$ l binding buffer, and stained with $5 \mu \mathrm{l}$ Annexin V conjugated with fluorescein isothiocyanate and $5 \mu \mathrm{l}$ propidium iodide at room temperature in the dark for $15 \mathrm{~min}$, and then immediately analyzed by a FACSCalibur System. The relative proportion of Annexin V-positive cells was determined using the CellQuest Pro software and counted as the percentage of apoptotic cells. The assay was performed in triplicate for three times.

Tumor formation assay in nude mice. Nude male mice that were 4 weeks old were obtained from the Laboratory Animal Center of Central South University (Changsha, China) and were maintained under specific pathogen-free conditions. For tumor formation experiment, $5 \times 10^{6}$ cells resuspended in $200 \mu \mathrm{l}$ of medium without serum were subcutaneously injected into the flanks of mice $(n=10$ mice each). The mice were monitored daily for palpable tumor formation, and tumor volume (in $\mathrm{mm}^{3}$ ) was measured by a Vernier caliper every 3 days and calculated by using the modified ellipse formula (volume $=$ length $\times$ width $^{2} / 2$ ). At the end of the experiments, the mice were killed by cervical dislocation, and tumors were excised, weighted, and embedded in paraffin for immunohistochemical staining.

To test the effect of miR-125b on in vivo NPC cells growth, $5 \times 10^{6}$ CNE2-IR cells were subcutaneously injected into the flanks of mice. When the xenograft volumes reached approximately $50 \mathrm{~mm}^{3}$, the transplanted mice were randomly divided into two groups ( $n=5$ mice each), $20 \mathrm{nmol}$ control or miR-125 antagomir (RiboBio) in $25 \mu$ l saline buffer was intratumorally injected into the tumor mass at multiple sites per mouse, and then tumor growth was monitored as above.

Western blot. Total proteins were exacted from cells as described previously by us. ${ }^{23}$ Nuclear and cytoplasmic proteins were prepared by using nuclear and cytoplasmic protein extraction kit (\#P0027, Beyotime) according to the manufacturer's instructions. An equal amount of protein in each sample was subjected to SDS-PAGE separation, followed by blotting onto a PVDF membrane. After blocking, blots were incubated with antibodies against A20 (ab92324, Abcam, Cambridge, MA, USA), p-IKK $\alpha / \beta$ (\#2078, CST), p-|кB $\alpha$ (\#2859, CST), p-p65(ReIA)
(\#3033, CST), IKK $\alpha$ (\#2682, CST), IкB $\alpha$ (\#4812, CST), p65(RelA) (\#4764, CST), or p53 (sc-47698, Santa Cruz, CA, USA) overnight at $4^{\circ} \mathrm{C}$, followed by incubation with HRP-conjugated secondary antibody (\#A24531 or \#A24512, Life Technologies) for $2 \mathrm{~h}$ at room temperature. The signal was visualized with an enhanced chemiluminescence detection reagent (Pierce). Tublin (ab15246, Abcom), $\beta$-actin (sc-1616, Santa Cruz) or Histone H3 (ab8580, Abcom) was simultaneously detected as a loading control. The assays were performed for three times.

Immunohistochemistry. Immunohistochemical staining of A20, p-p65(RelA), and $\mathrm{Ki}-67$ were performed on the formalin-fixed and paraffin-embedded tissue sections as described previously by us. ${ }^{46}$ In brief, after antigen retrieval tissue sections were incubated with antibodies against A20, p-p65(RelA), or -Ki-67 antibody (ab15580, Abcom) overnight at $4^{\circ} \mathrm{C}$, and then were incubated with biotinylated secondary antibody followed by avidin-biotin peroxidase complex. Finally, tissue sections were incubated with $3^{\prime}, 3^{\prime}$-diaminobenzidine (Sigma-Aldrich) and counterstained with hematoxylin. In negative controls, primary antibodies were replaced with a normal mouse or rabbit lgG. Known immunostaining-positive slides were used as positive controls.

The immunoreactions were evaluated independently by two pathologists as described previously by us. ${ }^{46} \mathrm{~A}$ staining score of $\leqslant 3$ was considered to be low expression; and a score of $>3$ was considered to be high expression. Quantitative evaluation of proliferation cells was done by examining the sections in five random microscopic fields and counting the number of Ki-67 positive nuclei among 1000 cancer cells under the light microscope. The rate of proliferation cells was expressed as positive cells per 100 cancer cells.

In situ detection of apoptotic cells. Terminal deoxynucleotidyl TUNEL was performed to detect apoptotic cells of formalin-fixed and paraffin-embedded tissue sections of xenograft tumors with in situ cell death detection kit (Roche Applied Science, Shanghai, China) according to the manufacturer's instructions. Quantitative evaluation of apoptotic cells was done by examining the sections in five random microscopic fields and counting the number of TUNEL-positive cancer cells among 1000 carcinoma cells under the light microscope. The apoptotic index was expressed as positive cells per 100 cancer cells.

Immunofluorescent staining. A total of $2 \times 10^{3}$ cells were plated into chamber slides and cultured with RPMI-1640 medium containing $10 \%$ FBS for $12 \mathrm{~h}$. Cells were fixed with $4 \%$ paraformaldehyde at room temperature for $15 \mathrm{~min}$, and then cell membranes were permeabilized with $0.1 \%$ Triton 100 at room temperature for $20 \mathrm{~min}$. Cells were washed with $1 \times$ PBS and blocked with $10 \%$ goat serum in PBS for $1 \mathrm{~h}$. Then cells were incubated with primary antibody against p-p65 (RelA) overnight at $4{ }^{\circ} \mathrm{C}$. After washing with $1 \times$ PBS for three times, cells were incubated with secondary antibodies conjugated with Alexa Fluor 594 (DI-1794, Vector, Burlingame, CA, USA) for $1 \mathrm{~h}$. The slides were washed three times with $1 \times$ PBS, counterstained with DAPI (Vector Laboratories), mounted and stored at $4{ }^{\circ} \mathrm{C}$ under dark conditions. Pictures were taken under a Leica DMI4000 microscope.

Statistical analysis. All experiments were carried out at least three times. Data were presented as the mean \pm S.D. Statistical analysis was conducted using SPSS 22.0 statistical software package. For comparisons between two groups, a Student $t$-test or $\chi^{2}$-test was used, and for analysis with multiple comparisons, oneway ANOVA test or Wilcoxon and Mann-Whitney test was used. Survival curves were obtained by using Kaplan-Meier method, and comparisons were made by using log-rank test. Univariate and multivariate survival analyses were conducted on all parameters by using Cox proportional hazards regression model. The Spearman rank correlation coefficient was used to determine the correlation between two parameters. All statistical tests were two-sided. $P$-values $<0.05$ were considered to be statistically significant.

Ethics statement. This study was approved by the ethics committee of Xiangya School of Medicine, Central South University, China. Written informed consent was obtained from all participants in the study. All animal experiments were undertaken in accordance with the Guide for the Care and Use of Laboratory Animals of Central South University, with the approval of the Scientific Investigation Board of Central South University.

\section{Conflict of Interest}

The authors declare no conflict of interest. 
Acknowledgements. This work was supported by the Major Program of National Natural Science Foundation of China (81230053), the National Basic Research Program of China (2013CB910502), and the National Natural Science Foundation of China (81472801, 81672687).

1. Schwarzenbach $\mathrm{H}$, Nishida N, Calin GA, Pantel K. Clinical relevance of circulating cell-free microRNAs in cancer. Nat Rev Clin Oncol 2014; 11: 145-156.

2. Calin GA, Croce CM. MicroRNA signatures in human cancers. Nat Rev Cancer 2006; 6: 857-866.

3. Volinia S, Calin GA, Liu CG, Ambs S, Cimmino A, Petrocca F et al. A microRNA expression signature of human solid tumors defines cancer gene targets. Proc Natl Acad Sci USA 2006; 103: 2257-2261.

4. Yin $\mathrm{H}$, Sun $\mathrm{Y}$, Wang $X$, Park J, Zhang Y, Li M et al. Progress on the relationship between miR-125 family and tumorigenesis. Exp Cell Res 2015; 339: 252-260.

5. Banzhaf-Strathmann J, Edbauer D. Good guy or bad guy: the opposing roles of microRNA 125b in cancer. Cell Commun Signal 2014; 12: 30.

6. Sun YM, Lin KY, Chen YQ. Diverse functions of miR-125 family in different cell contexts. J. Hematol Oncol 2013; 6: 6.

7. Shaham L, Binder V, Gefen N, Borkhardt A, Izraeli S. MiR-125 in normal and malignant hematopoiesis. Leukemia 2012; 26: 2011-2018.

8. Aggarwal BB, Sung B. NF-кB in cancer: a matter of life and death. Cancer Discov 2011; 1: 469-471.

9. Zhu DD, Zhang J, Deng W, Yip YL, Lung HL, Tsang CM et al. Significance of NF-kB activation in immortalization of nasopharyngeal epithelial cells. Int J Cancer 2016; 138: 117511-117585

10. Chou J, Lin YC, Kim J, You L, Xu Z, He B et al. Nasopharyngeal carcinoma-review of the molecular mechanisms of tumorigenesis. Head Neck 2008; 30: 946-963.

11. Verhoeven RJ, Tong S, Zhang G, Zong J, Chen Y, Jin DY et al. NF-kB signaling regulates expression of Epstein-Barr virus BART microRNAs and long noncoding RNAs in nasopharyngeal carcinoma. J Virol 2016; 90: 6475-6488.

12. Chung GT, Lou WP, Chow C, To KF, Choy KW, Leung AW et al. Constitutive activation of distinct NF-кB signals in EBV-associated nasopharyngeal carcinoma. J Pathol 2013; 231: 311-322.

13. Nakanishi C, Toi M. Nuclear factor-kappaB inhibitors as sensitizers to anticancer drugs. Nat Rev Cancer 2005; 5: 297-309.

14. Liu S, Chen ZJ. Expanding role of ubiquitination in NF-kB signaling. Cell Res 2011; 21: 6-21.

15. Wertz IE, O'Rourke KM, Zhou H, Eby M, Aravind L, Seshagiri $S$ et al. Deubiquitination and ubiquitin ligase domains of A20 downregulate NF-kappaB signalling. Nature 2004; 430 : 694-699.

16. Shembade N, Ma A, Harhaj EW. Inhibition of NF-kappaB signaling by A20 through disruption of ubiquitin enzyme complexes. Science 2010; 327: 1135-1139.

17. Hymowitz SG, Wertz IE. A20: from ubiquitin editing to tumour suppression. Nat Rev Cancer 2010; 10: 332-341.

18. Vereecke L, Beyaert R, Loo G. The ubiquitin-editing enzyme A20 (TNFAIP3) is a central regulator of immunopathology. Trends Immunol 2009; 30: 383-391.

19. Shembade N, Harhaj EW. Regulation of NF- $\mathrm{KB}$ signaling by the A2O deubiquitinase. Cell Mol Immuno 2012; 9: 123-130.

20. Kim SW, Ramasamy K, Bouamar H, Lin AP, Jiang D, Aguiar RC. MicroRNAs miR-125a and miR-125b constitutively activate the NF- $\mathrm{B}$ p pathway by targeting the tumor necrosisfactor alpha-induced protein 3 (TNFAIP3, A20). Proc Natl Acad Sci USA 2012; 109: 7865-7870.

21. Parisi C, Napoli G, Amadio S, Spalloni A, Apolloni S, Longone P et al. MicroRNA-125b regulates microglia activation and motor neuron death in ALS. Cell Death Differ 2016; 23: $531-541$.

22. Liu Z, Smith KR, Khong HT, Huang J, Ahn EE, Zhou M et al. miR-125b regulates differentiation and metabolic reprogramming of $T$ cell acute lymphoblastic leukemia by directly targeting TNFAIP3. Oncotarget 2016; 7: 78667-78679.

23. Qu JQ, Yi HM, Ye X, Zhu JF, Yi H, Li LN et al. MiRNA-203 reduces nasopharyngeal carcinoma radioresistance by targeting IL8/AKT signaling. Mol Cancer Ther 2015; 14 : 2653-2664

24. Compagno M, Lim WK, Grunn A, Nandula SV, Brahmachary M, Shen $Q$ et al. Mutations of multiple genes cause deregulation of NF-kappaB in diffuse large B-cell lymphoma. Nature 2009; 459: 717-721.

25. Kato M, Sanada M, Kato I, Sato Y, Takita J, Takeuchi K et al. Frequent inactivation of TNFAIP3 in B-cell lymphomas. Nature 2009; 459: 712-716.

26. Braun FC, Grabarczyk P, Möbs M, Braun FK, Eberle J, Beyer M et al. Tumor suppressor TNFAIP3 (A20) is frequently deleted in Sézary syndrome. Leukemia 2011; 25: 1494-1501.

27. Schmitz R, Hansmann ML, Bohle V, Martin-Subero Jl, Hartmann S, Mechtersheimer G et al. TNFAIP3 (A20) is a tumor suppressor gene in Hodgkin lymphoma and primary mediastinal B cell lymphoma. J Exp Med 2009; 206: 981-989.
28. Honma K, Tsuzuki S, Nakagawa M, Tagawa H, Nakamura S, Morishima Y et al. TNFAIP3/ A20 functions as a novel tumor suppressor gene in several subtypes of non-Hodgkin lymphomas. Blood 2009; 114: 2467-2475.

29. Troppan K, Hofer S, Wenzl K, Lassnig M, Pursche B, Steinbauer E et al. Frequent down regulation of the tumor suppressor gene TNFAIP3 in multiple myeloma. PLoS One 2015; 10 : e0123922.

30. Liang $Y, X u R Z$, Zhang L, Zhao XY. Berbamine, a novel nuclear factor kappaB inhibitor, inhibits growth and induces apoptosis in human myeloma cells. Acta Pharmacol Sin 2009; 30: 1659-1665.

31. Langsch S, Baumgartner U, Haemmig S, Schlup C, Schäfer SC, Berezowska S et al. miR-29b mediates NF- $\mathrm{kB}$ signaling in KRAS-induced non-small cell lung cancers. Cancer Res 2016; 76: 4160-4169.

32. Hjelmeland AB, Wu Q, Wickman S, Eyler C, Heddleston J, Shi Q et al. Targeting TNFAIP3 decreases glioma stem cell survival and tumor growth. PLOS Biol 8: e1000319.

33. Bellai AC, Olson JJ, Yang X, Chen ZJ, Hao C. TNFAIP3 ubiquitin ligase- mediated polyubiquitination of RIP1 inhibits caspase-8 cleavage and TRAIL- induced apoptosis in glioblastoma. Cancer Discov 2012; 2: 140-155.

34. Wang CM, Wang Y, Fan CG, Xu FF, Sun WS, Liu YG et al. miR-29c targets TNFAIP3, inhibits cell proliferation and induces apoptosis in hepatitis $B$ virus-related hepatocellular carcinoma. Biochem Biophys Res Commun 2011; 411: 586-592.

35. Zhang L, Ding Y, Yuan Z, Liu J, Sun J, Lei F et al. MicroRNA-500 sustains nuclear factor- $\mathrm{kB}$ activation and induces gastric cancer cell proliferation and resistance to apoptosis. Oncotarget 2015; 6: 2483-2495.

36. Selbach M, Schwanhausser B, Thierfelder N, Fang Z, Khanin R, Rajewsky N. Widespread changes in protein synthesis induced by microRNAs. Nature 2008; 455: 58-63.

37. Busch S, Auth E, Scholl F, Huenecke S, Koehl U, Suess B et al. 5-lipoxygenase is a direct target of miR-19a-3p and miR-125b-5p. J Immunol 2015; 194: 1646-1653.

38. Wu JG, Wang JJ, Jiang X, Lan JP, He XJ, Wang HJ et al. MiR-125b promotes cell migration and invasion by targeting PPP1CA-Rb signal pathways in gastric cancer, resulting in a poor prognosis. Gastric Cancer 2015; 18: 729-739.

39. Wang YD, Cai N, Wu XL, Cao HZ, Xie LL, Zheng PS. OCT4 promotes tumorigenesis and inhibits apoptosis of cervical cancer cells by miR-125b/BAK1 pathway. Cell Death Dis 2013 4: e760.

40. Amir S, Ma AH, Shi XB, Xue L, Kung HJ, Devere White RW. Oncomir miR-125b suppresses p14 (ARF) to modulate p53-dependent and p53-independent apoptosis in prostate cancer PLoS One 2013; 8: e61064.

41. Ahuja D, Goyal A, Ray PS. Interplay between RNA-binding protein HuR and microRNA-125b regulates p53 mRNA translation in response to genotoxic stress. RNA Biol 2016; 13: $1152-1165$.

42. Sun $Y, Y i H$, Yang $Y, Y u ~ Y$, Ouyang $Y$, Yang $F$ et al. Functional characterization of $p 53$ in nasopharyngeal carcinoma by stable shRNA expression. Int J Oncol 2009; 34: 101710-101727.

43. Wu N, Lin X, Zhao X, Zheng L, Xiao L, Liu J et al. MiR-125b acts as an oncogene in glioblastoma cells and inhibits cell apoptosis through p53 and p38MAPK-independent pathways. Br J Cancer 2013; 109: 2853-2863.

44. Shanmugaratnam K, Sobin LH. The World Health Organization histological classification of tumours of the upper respiratory tract and ear. A commentary on the second edition. Cancer 1993: 71: 2689-2697.

45. Pan J, Xu Y, Qiu S, Zong J, Guo Q, Zhang Y et al. A Comparison between the Chinese 2008 and the 7th edition AJCC staging systems for nasopharyngeal carcinoma. Am J Clin Oncol 2015; 38: 189-196.

46. Feng XP, Yi H, Li MY, Li XH, Yi B, Zhang PF et al. Identification of biomarkers for predicting nasopharyngeal carcinoma response to radiotherapy by proteomics. Cancer Res 2010; 70 : 3450-3462.

(i) Cell Death and Disease is an open-access journal published by Nature Publishing Group. This work is licensed under a Creative Commons Attribution 4.0 International License. The images or other third party material in this article are included in the article's Creative Commons license, unless indicated otherwise in the credit line; if the material is not included under the Creative Commons license, users will need to obtain permission from the license holder to reproduce the material. To view a copy of this license, visit http://creativecommons.org/licenses/by/4.0/

(C) The Author(s) 2017

Supplementary Information accompanies this paper on Cell Death and Disease website (http://www.nature.com/cddis) 